\title{
NGS for the diagnosis of autoinflammatory diseases: the experience of Montpellier
}

\author{
G Sarrabay, G Tachon, D Mechin, I Touitou* \\ From 8th International Congress of Familial Mediterranean Fever and Systemic Autoinflammatory Diseases \\ Dresden, Germany. 30 September - 3 October 2015
}

\section{Introduction}

Monogenic autoinflammatory diseases present with overlapping clinical features such as recurrent fever, biological inflammation, abdominal pain, arthritis, and sometimes disabling complications. Over 30 genes have been confirmed or hypothesized as causing diseases. Sanger sequencing is the gold-standard approach for genetic diagnosis, but cannot be exhaustive.

\section{Objectives}

We aimed at offering a quick and efficient service for these genetically heterogeneous disorders through NGS sequencing of all published and candidate $(\mathrm{N}=32)$ autoinflammatory genes.

\section{Patients and methods}

34 patients were selected on clinical grounds by members of the French reference center for autoinflammatory diseases (CeReMAI). 9 of them were considered positive control samples as they had previously known variants (by Sanger sequencing) and were run in parallel for methodological validation.

A custom panel of $108 \mathrm{~kb}$ was designed to capture the genomic regions of interest using the Illumina Nextera Rapid Capture enrichment DNA preparation kit. MicroV2 chip were then loaded with 12 samples on a MiSeq equipment for multiparallel sequencing. Annotation and filtering were performed with Seqnext software (JSI), and annotation was performed with Alamut Visual (Interactive Biosoftware).

\section{Results}

We obtained a $545 \mathrm{X}$ average coverage and only 2 exons were not captured. Minimal coverage was 40X. Results were concordant in $100 \%$ of the cases with

CHRU Montpellier, Montpellier, France 